\author{
Voix plurielles \\ Volume 5, Numéro 1 : mai 2008
}

Élise Lepage

\title{
De la domestication : la maison - le corps - le livre dans la poésie de Pierre Morency et de Pierre Nepveu
}

Citation MLA : Élise Lepage. «De la domestication : la maison - le corps - le livre dans la poésie de Pierre Morency et de Pierre Nepveu.» Voix plurielles 5.1 (mai 2008).

(C) Voix plurielles, revue électronique de l'APFUCC 2008. 


\title{
De la domestication : la maison - le corps - le livre dans la poésie de Pierre Morency et de Pierre Nepveu
}

\author{
Élise Lepage \\ Université de Colombie-Britannique
}

Mai 2008

\section{Résumé}

Cet article propose une lecture des recueils de poèmes de Pierre Morency et Pierre Nepveu à la lumière de l'ouvrage Habit Habitat Habitus de Carole Simard-Laflamme paru aux éditions d'art Le Sabord. Habit Habitat Habitus rassemble des réflexions d'artistes ou de chercheurs issus de domaines très différents sur les analogies existantes entre espace corporel, espace référentiel et manière d'être, comportement du sujet. Néanmoins, le triptyque du titre semble masquer un quatrième aspect pourtant fondamental, évident du texte: celui de l'espace du livre. En effet, l'œuvre de Simard-Laflamme s'apparente presque autant à un objet artistique qu'à un livre au sens traditionnel. Les pages compilent textes, photographies, images plus ou moins détournées, schémas, etc., et ce dans un jeu de couleurs et de formes qui ne laisse pas l'œil indifférent. Par le biais de l'analogie, l'auteure fait apparaître des correspondances entre espace corporel et espace d'habitation, espace fictif et référentiel, celles-là même que développent les textes de Morency et de Nepveu.

Comment dès lors cette analogie entre corps, habitat, espaces référentiel et fictif et espace livresque se décline-t-elle pour ébaucher une subjectivité toute empreinte de son rapport au lieu, à l'acte d'habiter? Il apparaît en outre que ces analogies permettent de se familiariser avec l'inconnu, de domestiquer l'étrangeté. La question n'est donc plus celle de l'habitation du monde en poésie, mais de la domestication de l'étrangeté de ce monde. Comment le Je lyrique aménage le poème à l'image de ces figures fondamentales que sont le corps, le vêtement ou la maison?

Habit Habitat Habitus de Carole Simard-Laflamme, paru en 2002 aux éditions d'art Le Sabord, se présente comme un livre indomptable.

Figure 1 : Table des co-auteurs de Habit Habitat Habitus

Figure 2 : Table des matières de Habit Habitat Habitus 
Cet objet textuel protéiforme, difficilement totalisable, rassemble des réflexions d'artistes ou de chercheurs issus de domaines très différents sur les analogies existantes entre espace corporel, espace référentiel et manière d'être. Néanmoins, le triptyque du titre semble masquer un quatrième aspect pourtant fondamental et évident du texte : celui de l'espace du livre. En effet, l'œuvre de Simard-Laflamme s'apparente presque autant à un objet artistique qu'à un livre au sens traditionnel. Les pages compilent textes, photographies, images plus ou moins détournées, schémas, etc.

Figure 3 : Présentation d'Habit Habitat Habitus

par l'éditeur en quatrième page de couverture

L'auteure entreprend de déceler les analogies, les correspondances existantes entre espace corporel et espaces d'habitation, ses investigations ne manquant finalement pas de remettre en question l'espace textuel (autrement dit, la façon dont le texte est agencé sur la page) et l'espace livresque (celui du livre comme objet matériel pour le lecteur). Cette entreprise se situe donc dans le sillage de plusieurs critiques littéraires et théoriciens ${ }^{1}$ du langage qui, depuis un demisiècle déjà, ont souligné l'importance de la spatialité du langage et du texte littéraire, alors que les études littéraires s'intéressent traditionnellement aux relations entre temps et écriture. Dans un article paru en 1964, Foucault constatait ainsi :

Écrire, pendant des siècles, s'est ordonné au temps. . . S'adressant ou non au passé, se soumettant à l'ordre des chronologies ou s'appliquant à le dénouer, l'écriture était prise dans une courbe fondamentale qui était celle du retour homérique, mais celle aussi de l'accomplissement des prophéties juives. Alexandrie, qui est notre lieu de naissance, avait prescrit ce cercle à tout le langage occidental: écrire, c'était faire retour, c'était revenir à l'origine, se ressaisir du premier moment; c'était être de nouveau au matin.

Le $\mathrm{XX}^{\mathrm{e}}$ siècle est peut-être l'époque où se dénouent de telles parentés. Le retour nietzschéen a clos une bonne fois la courbe de la mémoire platonicienne, et Joyce refermé celle du récit homérique. Ce qui ne nous condamne pas à l'espace comme à une seule autre possibilité, trop longtemps négligée, mais dévoile que le langage est (ou, peut-être, est devenu) chose d'espace. (407)

L'enjeu de cette réflexion met ainsi en place un double questionnement: d'une part, comment les formes primordiales relevées par Simard-Laflamme permettent-elles d'appréhender le monde? En quoi constituent-elles un réservoir à la fois minimal et inépuisable de métaphores littéraires pour représenter le monde? D'autre part, comment l'espace du texte peut-il être travaillé afin de lui aussi fonctionner comme une image de ces formes primordiales? Autrement dit, la maison, le vêtement ou le corps feraient image non pas seulement au niveau de la mimèsis, mais aussi au niveau le plus matériel de l'écriture (de la spatialité inhérente au langage à la disposition du texte sur la page ou encore la présentation matérielle du livre). 
À la lumière de l'œuvre aux multiples facettes de Carole Simard-Laflamme, cette réflexion initie un bref parcours à travers les œuvres poétiques de Pierre Morency et de Pierre Nepveu, la question du lieu à habiter et à écrire occupant une place prépondérante chez ces deux poètes québécois contemporains. Tous deux ont commencé à publier à la fin de la décennie 1960, Morency dans un sillage plus ou moins proche de la poésie du pays, Nepveu dans les marges des avant-gardes formalistes. Quoique de manière différente de chez Simard-Laflamme, leurs œuvres depuis une vingtaine d'années interrogent et superposent elles aussi ces formes élémentaires que sont la maison, le vêtement ou le corps afin de donner forme au cosmos. Comment dès lors l'instance poétique appréhende-t-elle le réel à travers ces formes primordiales qui permettent de reformuler la question de l'habitation du monde en poésie?

La maison, le vêtement, mais aussi la peau (comme enveloppe du corps) fonctionnent comme des formes gigognes qui permettent de concevoir le réel. D'une certaine manière, le langage peut également être perçu comme l'une de ces enveloppes, devenant ainsi une sorte de "demeure mobile » que la poésie s'essaie d'habiter. Mais la poésie pose-t-elle la bonne question lorsqu'elle s'interroge sur l'acte d'habiter? Cet article insistera dans un dernier temps sur le concept de domestication, peut-être plus productif et mieux à même de rendre compte de la tension entre étrangeté et familiarité qui travaille l'espace textuel et l'espace livresque.

\section{Habitat - Habit - Peau - Mots : des formes gigognes pour concevoir le réel}

De l'extérieur vers l'intérieur, du monde au moi, la maison, le vêtement, la peau sont des enveloppes successives, gigognes; tel est le point initial de l'entreprise de Carole SimardLaflamme dans Habit Habitat Habitus : «avant tout, avant la forme : l'informe. Presque rien. . . Bulle du cerveau. Peau de l'écorché, tombante. Membrane, robe sans corps. Presque tout. Viennent alors le squelette, la charpente, le corps : la forme. » (9). Maison, vêtement et peau habillent progressivement l'homme qui en retour, se doit d'apprendre à les habiter au sens plein pour véritablement être. Le mur, le tissu, la peau sont ce par quoi l'être prend forme et se détache du reste du monde; ils créent un espace minimal de l'intime qui résiste à l'étrangeté du monde environnant. Ils dessinent une forme plus ou moins précise de l'être, lui donnent une silhouette. Face à un monde instable, dilué, Pierre Nepveu affirme le contour des êtres et des choses :

combien le monde nous soutient peu, nos corps trouvant (entre la terre et les oiseaux) cet équilibre qui se brise parfois par malice dans l'aube tremblante, où chaque objet et chaque être se redessinent pourtant plus clairs et plus fermes (Romans-fleuves, 18).

Simard-Laflamme décrit ces formes qui emboîtent successivement le Je dans le monde. Elles fonctionnement comme des images, des schémas élémentaires mis en œuvre pour donner forme à l'espace humain: «l'histoire et ses ramifications nous rapportent de nombreux exemples où l'homme a étendu son royaume à l'image et aux correspondances des parties de son corps : le corps comme modèle organisationnel. » (31). Elle cite en guise d' exemple, images à l'appui, des 
chapeaux de paille et huttes tressées, casques guerriers de métal et forteresses de pierres, mitres brodées de fils d'or et architectures religieuses richement ornées, chapeaux en hauteur et tours des châteaux, hennins allongés et verticalités gothiques, coiffures gonflées et coupoles amplifiées. "L'individu s'est logé comme il s'est vêtu. » (39).

Figure 4 : Analogies entre vêtement et habitation à travers l'Histoire, Habit Habitat Habitus 98.

Les poèmes de Morency offrent maintes images analogues: sous l'effet du froid, des «maisons s'étirent et craquent des os » (Torrentiel 218) et dans Lieu de naissance, «la peau des maisons avait cédé sous les couteaux du nordet » (167). Maison, vêtement et peau s'échangent mutuellement leurs formes afin d'organiser le réel, c'est-à-dire de le rendre habitable.

Mais par-delà ce qui s'apparente presque à des lieux communs, n'est-ce pas là également une manière de décrire par image le processus qu'implique toute écriture : celui de mettre en mots, c'est-à-dire de rendre concevable ce qui de prime abord se présente chaotiquement, tel le monde, le vécu humain, les relations aux autres, ou encore la mort? Écrire, tout comme le fait d'habiter ces différentes épaisseurs, consiste à donner forme au réel, à le rendre scriptible et lisible. Cette réflexion se propose donc de prolonger celle de Carole Simard-Laflamme en faisant apparaître à quel point le langage fonctionne lui aussi comme l'une de ces couches successives, permettant au sujet d'appréhender le monde et de représenter le réel.

Or, bien des auteurs et des philosophes éprouvent le langage comme la demeure la plus intime qui soit, plus proche encore du moi que ne l'est la peau. En poésie, le langage est souvent métaphorisé par la maison. Une maison au toit souvent rouge chez Morency, tels qu'en témoignent ces exemples tirés de différents recueils : « J'écris l'étroite maison rouge. . . (Effets personnels 253); « sur la ligne de l'horizon / une maison au toit rouge / représente la vie actuelle du poète » (Douze jours dans une nuit 243), à moins que ce ne soit la vôtre : « - sans partir de chez lui il est venu chez vous il fouille dans les trous que vous faites en parlant. » (Torrentiel 220). Mais cette métaphore de la maison est ambivalente dans la mesure où elle combine deux sèmes : celui de l'habitation et celui de la sédentarité. Elle n'est à cet égard qu'à moitié fidèle pour représenter le langage : si la question est bien d'habiter le langage comme on habite une maison, celui-ci en revanche est tout sauf enracinement, sédentarité; ma langue, mes mots forment une demeure mobile qui me suit partout; tel est l'une des belles réflexions de Derrida :

Que nomme en effet la langue, la langue dite maternelle, celle qu'on emporte avec soi, celle qui nous emporte aussi de la naissance à la mort? Ne figure-t-elle pas le chez-soi qui ne nous quitte jamais? Le propre ou la propriété, le phantasme au moins de propriété qui, au plus près de notre corps. . . donnerait lieu au lieu le plus inaliénable, à une sorte d'habitat mobile, un vêtement ou une tente? Ladite langue maternelle, ne serait-ce pas une sorte de seconde peau qu'on porte sur soi, un chez-soi mobile? Mais aussi 
un chez-soi inamovible puisqu'il se déplace avec nous?. . . La langue résiste à toutes les mobilités parce qu'elle se déplace avec moi. Elle est la chose la moins inamovible, le corps propre le plus mobile qui reste la condition stable, mais portable, de toutes les mobilités. (85).

Le langage, les mots de ma langue maternelle réalisent ce paradoxe d'être à la fois ce qui m'est le plus ancré, le plus propre, mais aussi ce que je ne maîtrise pas, ce qui me poursuit, m'enveloppe et à quoi je n'échappe jamais vraiment. En cela, le langage s'apparente au soulier, attribut essentiel de la figure du poète chez Morency: «Le soulier, c'est le point de contact que nous avons en permanence (ou presque) avec le sol, avec la terre, avec le monde. » (La Vie entière 142). Le soulier et le langage fonctionnent ainsi comme des racines mobiles, une présence au réel dont nous ne nous départons jamais, où que nous soyons. Le langage est marqué du sceau de l'intimité absolue, comme étant notre attribut le plus personnel. «La peau, dit le poète, est ce que nous avons de plus profond » (L'œeil américain 113), écrit encore Morency en jouant du paradoxe entre superficialité et profondeur. Il pourrait en être dit tout autant du langage, enveloppe extérieure qui nous permet de nous exprimer et de nous ouvrir à autrui, mais aussi profond terreau qui définit notre façon d'être au monde. Le langage s'inscrit alors dans la continuité de notre énumération : habitat, habit, peau, mots. Face à un «cosmos brisé », Nepveu se rétracte ainsi sur son

seul pouvoir d'emporter au loin sa carrure, sa maison, ses mots, leur enlever toute forme (Romans-fleuves 81).

Il énumère ici l'emboîtement de formes élémentaires précédemment identifiées (corps, maison, mots), formes qui permettent d'appréhender le monde, qui conduisent de l'étrangeté du réel à l'intimité du moi. Mais ces vers posent aussi la question de l'habitation du langage comme « demeure mobile».

\section{Habiter la « demeure mobile » du langage}

On connaît la prégnance de la question de l'habitation en littérature, particulièrement en poésie, dans le sillage de la pensée d'Heidegger. L'essai «Bâtir Habiter Penser » rappelle que «l'espace est essentiellement ce qui a été «ménagé », ce que l'on a fait entrer dans sa limite » (183), c'est-à-dire ce qui a reçu une forme. Étymologiquement, l'allemand lie essence et habitation: «le vieux mot bauen, auquel se rattache bin, nous répond «je suis », «tu es », veulent dire : j'habite, tu habites. La façon dont tu es et dont je suis, la manière dont nous autres hommes sommes sur terre est le buan, l'habitation.» (Heidegger 173). Être et habiter désigneraient donc un seul et même acte. Or, c'est un truisme que de dire que le poète est poète (essence) en tant qu'il habite la demeure du langage (habitation). Et comme tout truisme, il est bon de le remettre en question; car comment habiter réellement le langage s'il fonctionne comme une «demeure mobile », oxymore qui désigne son ambiguïté? En fait, c'est la question même de l'habitation que nous proposons de remettre en cause. En français, l'habitation est liée à l'avoir 
(habere). Or, qui peut prétendre posséder souverainement le langage? Nul ne s'y risquerait, et surtout pas le poète. Le monde est là, mais il ne m'appartient pas encore complètement. Faut-il même souhaiter qu'il m'appartienne un jour? Et serait-ce vraiment là le propos des entreprises poétiques de Nepveu et de Morency, que de s'approprier le monde au sens fort? Leurs poèmes suggèrent une conception moins totalitaire, moins exclusive. Il semble en effet que chez ces deux poètes, ce sont des tentatives de «domestication » qui sont à l'œuvre, concept plus prudent que nous suggérons de substituer à celui d" " habitation ».

Que signifie le fait de domestiquer? Sans doute moins vouloir s'approprier que chercher à apprivoiser, à appréhender, à rendre intelligible, familier. À l'origine, domes-tiquer, c'est faire rentrer dans la forme primordiale de la maison, incorporer au monde connu (ici, les mots mêmes permettent de retrouver les formes fondamentales de la maison et du corps). Au contraire de l'habitation qui suppose un état de fait, une prise de possession, la domestication ${ }^{1}$ est processus. Elle invite à l'approche lente, patiente, parfois laborieuse; elle nécessite une fréquentation assidue. Cette démarche semble relever d'une fascination envers le monde, totalité qui nous échappe dans son ensemble et dans ses détails, mais ne cesse de nous interroger : "Toucher au monde restait infiniment / permis et désirable, » (Romans-fleuves 71) écrit Pierre Nepveu. Ce «toucher au monde » marque la volonté d'une approche. Il n'est plus question d'une immatérielle habitation, mais d'une domestication bien concrète. Car domestiquer, tenter d'apprivoiser, n'estce pas aussi ce que nous mettons à l'œuvre en permanence au quotidien face à l'inconnu, à l'étrangeté? Nous trouvant dans un lieu où nous n'avons jamais été, rencontrant une personne qui nous est inconnue, nous essayons tout aussitôt de cerner ces expériences de l'étrangeté en mobilisant nos catégories de pensée préexistantes, celles que nous maîtrisons. Tout l'enjeu de la découverte, de la rencontre, tient justement à ce glissement auquel ces situations nous invitent : l'Autre, l'étrangeté nous convient à augmenter, à réinventer constamment nos schèmes de pensée. Ils nous permettent de domestiquer, autrement dit de nous familiariser avec des concepts que nous ignorions jusqu'alors. Cette familiarisation progressive permet l'élargissement, l'approfondissement progressif de notre façon d'être au monde. Elle s'effectue exemplairement à travers les schèmes de pensée fondamentaux relevés par Carole Simard-Laflamme : la maison, le vêtement ou la peau. Transposée à un niveau plus conceptuel, le langage devient alors un autre de ces modèles².

$\mathrm{Si}$ nous éprouvons couramment dans notre expérience du monde ce processus de domestication, n'est-il pas pour autant voué à l'échec dans la sphère littéraire? Domestiquer désigne le passage de l'étrangeté au familier, ne se situe-t-il pas aux antipodes de ce qui fonde tout projet poétique? Certes, écrire un poème, c'est organiser, donner forme à un microcosme. Toute mise en mots est une mise en ordre possible du monde. Par-delà, écrire consiste à remettre en question le familier, les évidences, tout ce que l'habitude use, mais aussi et peut-être surtout les mots couramment utilisés pour décrire ce que nous appréhendons. Si un premier stade de la création consiste donc à domestiquer le monde par le langage, il s'agit ensuite de restituer au texte l'irréductible étrangeté du monde par la mise en œuvre d'un langage qui permet précisément d'amorcer un glissement des catégories de pensée, tant celles de l'auteur que du lecteur. Autrement dit, c'est par le langage, ressenti nous l'avons vu comme forme la plus intime, la plus proche du moi, que réapparait l'étrangeté.

\section{Le livre, un espace sous tension}


Si la domestication désigne le passage de l'étrangeté au familier, elle semble s'inscrire dans une dynamique inverse à celle qui fonde tout projet poétique. Écrire, c'est certes organiser, donner forme à ce qui n'en a pas toujours dans le monde référentiel. Le livre fonctionne alors comme un microcosme qui s'essaie à mettre en page (comme on «met en boîte ») l'étrangeté du monde. Mais cette étrangeté ne tarde jamais à réapparaître au détour d'un vers. Le sujet lyrique de Romans-fleuves de Pierre Nepveu s'observe écrire avec effroi :

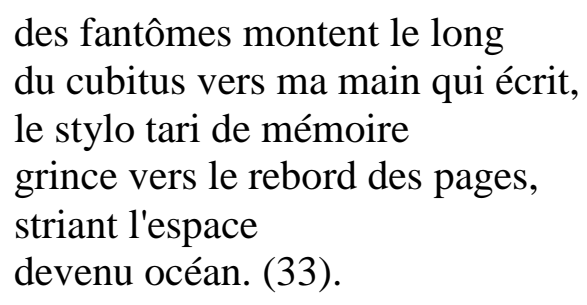

L'étrangeté ressurgit au cœur de l'identité et se propage jusque sur le support de l'écriture; tel ce stylo qui hésite dans les marges du texte, comme s'il n'osait prendre la pleine mesure de l'espace de la page et des vertiges qu'il contient. Un étonnement comparable, quoique peut-être plus jubilatoire qu'inquiet, se retrouve chez Pierre Morency: «La page blanche est pleine de mondes qui cherchent à vivre, elle grouille de caractères qui ne demandent qu'à dévoiler, » (Les Paroles qui marchent dans la nuit 19). Dans le même recueil, il s'émeut quelques pages plus loin devant «la beauté de ce paysage que nous ne pourrons jamais habiter» (27). Ce paysage n'est pas seulement celui qui s'offre aux yeux du Je, mais c'est aussi le paysage livresque qui se trouve sous les yeux du lecteur, la double page du livre ouvert. Chez les deux poètes, le livre comme possibilité de donner forme au monde ne va pas sans une réflexion métatextuelle sur le livre comme support, comme lieu de l'écrit, rejoignant en cela le projet de Carole SimardLaflamme.

Une double interrogation portant sur l'étrangeté du lieu et du livre (tous deux à domestiquer) fait alors jour. On la retrouve dans Lignes aériennes, recueil consacré à la déchéance de l'aéroport de Mirabel :

ici en ce bout du monde qui frôle la parodie, en cette clairière où les petites forêts d'érables viennent s'agenouiller pour adorer l'espace vacant d'un parc industriel, ici où vaquent des âmes errantes qui n'ont pas perdu leur attache et qui restent à mâcher de la fougère dans les sous-bois cristallisés, je ne sais plus vraiment si tout ceci a un sens et il se pourrait

$$
\begin{aligned}
& \text { que ce soit mieux } \\
& \text { ainsi, il se pourrait } \\
& \text { que je ne sois là } \\
& \text { que pour témoigner }
\end{aligned}
$$

de ce qui reste, au bout d'une autoroute qui se termine dans les ronces et le chiendent - en d'autres temps le monde était une grille heureuse, une toile dont chaque fil faisait synapse ou cordon, en d'autres temps l'écheveau fabuleux des routes nous déportait sans 
douleur vers la rencontre de nous-mêmes. (Nepveu, Lignes aériennes 77).

Ce que dit et donne à voir ce poème de Nepveu jusque dans sa disposition graphique, c'est la pauvreté d'un ici dévasté par la postmodernité. L'étranglement du texte amorce pourtant un possible changement de point de vue (introduit par le conditionnel «il se pourrait»); mais l'imaginaire du déchet, de l'épars si caractéristique de la pensée de Nepveu s'oppose de plein fouet à la mémoire du lieu, autrefois lisible, regorgeant de sens dans les liens, les analogies qui le maintenaient. Le paysage que donne à voir les environs de Mirabel n'a donc plus rien de cette «grille heureuse », tout comme la mise en page de ce poème se rebiffe contre une disposition traditionnelle du poème (des strophes carrées ou rectangulaires closes, « grillagées »).

De façon apparentée, le dernier recueil de Pierre Morency, À l'heure du loup (2002), constitué essentiellement de proses, reproduit graphiquement une pierre tombale de l'Abbaye de Westminster où « les plaques tombales. . . sont posées à même le carrelage où les visiteurs sont bien obligés pour circuler de les fouler du pied. » (121). Le Je décrit:

Je ressens alors, à mesure que je lis, une étrange impression. Celle de sentir une main sourdre du sol et saisir le bas de mon pantalon. Que se passe-t-il donc?

\author{
ANTHONY TROLLOPE \\ $1815-1882$ \\ NOW I STRETCH MY HAND \\ AND FROM THE FURTHER SHORE I BID \\ ADIEU TO ALL WHO HAVE CARED TO \\ READ ANY AMONG THE MANY WORDS \\ I HAVE WRITTEN
}

(122).

Cet extrait est suivi de près par l'évocation du Mémorial du 13 mai 1933 à Berlin :

Ce monument est invisible à qui ne sait pas regarder où il marche : il se présente sous la forme d'un verre épais, transparent, intégré au sol. À travers cette fenêtre inattendue, on discerne, en bas, dans une pièce nue, un alignement d'étagères vides. C'est à cet endroit précis, $\mathrm{qu}[\mathrm{e}]$... les nazis détruisirent en autodafé des milliers de livres d'auteurs disgraciés par le régime: des poètes et scientifiques juifs, communistes ou pacifiques. On dit que pour cette tâche les policiers furent secondés par les étudiants de l'université toute proche, qui sortaient par pleines brassées les volumes de la bibliothèque. (123).

En guidant le regard du visiteur et du lecteur vers le sol, Morency nous convie dans ces lieux de mémoire, lieux de mort de la littérature. La localisation, celle de l'enracinement de l'Histoire, est primordiale. À travers les modestes dimensions du livre de poésie et les virtualités limitées du 
langage écrit, Pierre Morency fait son possible pour restituer l'étrangeté dérangeante que créent l'architecture et la disposition de ces lieux. Ses mots disent ici leur incapacité à reproduire graphiquement et sémantiquement tout ce que représentent (symboliquement, mais aussi ce que donnent à voir) ces lieux de mémoire et leur agencement. Ainsi, le langage permet ultimement le retour de l'étrangeté du réel. Par son entremise, l'entreprise formelle du poème ne réussit jamais totalement: les mots par lesquels advient la forme du poème sont ceux-là mêmes qui réintroduisent l'irreprésentable, l'informe, le chaos du réel.

La domestication ne serait ainsi qu'une étape dans la création littéraire, étape qui correspondrait au difficile passage à la mise en mots. Par son inadéquation à exprimer le réel, le langage mis en œuvre (et cette expression peut se lire littéralement dans le sens de «mis dans un livre ») fait ressurgir l'inadéquation de nos catégories de pensées, de nos mots face au monde. L'évidence et la banalité de ce propos retrouve toute sa prégnance face au livre de SimardLaflamme qui joint à l'étrangeté du langage, l'étrangeté de l'objet livre. Habit Habitat Habitus cède son caractère d'objet quotidien, banal pour s'auréoler d'étrangeté, celle de ses procédés typographiques et plastiques. Cette remise en question du livre transforme ce qui désormais apparaît comme un objet commun, familier, sériel dans nos sociétés en un objet particulier, étrange, espace d'écriture au sens fort. Ce livre-objet dit alors dans sa matérialité même l'impossible domestication du réel, du livre et semble réaliser cette " autre idée d'un autre livre » que Barthes appelait de ses vœux et qui consistait à « rompre matériellement le fil de la phrase par des alinéas disparates, égaler en importance un mot et une phrase », ceci afin de concourir « à la destruction même du Livre », ce «Livre-Objet [qui] se confond matériellement avec le LivreIdée, la technique d'impression avec l'institution littéraire, en sorte qu'attenter à la régularité matérielle de l'œuvre, c'est viser l'idée même de littérature. En somme, les formes typographiques sont une garantie du fond. » (175) En filigrane de ces lignes, se lit le même projet de rompre avec une conception harmonieuse du monde dont la littérature serait la «grille heureuse » qui permettrait de le déchiffrer. Si par sa forme et son existence même, le livre est une domestication possible du monde, les fissures du langage qu'il met en œuvre le lézardent de toutes parts, font ressortir l'irréductible étrangeté du monde et des mots eux-mêmes. Dans les poèmes de Pierre Nepveu et de Pierre Morency, la mise en mots et la mise en page produisent un effet de mise à distance comparable qui fait réapparaître l'étrangeté. Par leur inadéquation à exprimer le réel, les mots de ces textes en viennent à pointer leur propre étrangeté et celle du livre, microcosme peut-être devenu trop familier.

Chez Pierre Morency, le poème précède ou suit toujours de peu la naissance. Le questionnement porte sur l'apparition de cette nouvelle vie et la forme par laquelle elle advient. Chez Pierre Nepveu au contraire, ses poèmes se situent toujours dans un «après » : il ne s'agit que rarement de donner un contour, mais plutôt de constater la dislocation des formes. Domestiquer ce réel en miettes, c'est tenter cet impossible rapiéçage de fragments, lui donner forme à travers l'habitat, l'habit, la peau ou les mots, ces formes perméables et échangeables qui permettent au sujet de domestiquer le monde et son étrangeté inhérente. Le langage, forme la plus proche du moi, se révèle pourtant la moins domesticable et ramène inexorablement à l'étrangeté du monde.

Dans ce contexte, le livre devient un microcosme sous tension permanente entre l'exclusion provisoire de l'étrangeté (le livre donne forme au monde) et le retour de cette étrangeté par 
l'entremise du langage. L'inadéquation des mots au réel renforcée par différents procédés typographiques et plastiques viennent rappeler que si les poètes habitent en permanence le langage, ils ne le domestiquent jamais vraiment; tout au plus «squattent »-ils cette «demeure mobile » des mots, à la façon des nomades. Par ce resurgissement de l'étrangeté, le livre donne bientôt autant à voir qu'à lire («dessiner un poème, nous dit Morency, c'est pour voir que les gens lisent » (Effets personnels 251)). Habit Habitat Habitus en est à la fois une illustration possible, mais aussi une invitation à une réflexion métalittéraire de première importance qui se situe au croisement des arts poétiques et visuels, à replacer dans un contexte contemporain de diversification des supports des écrits littéraires.

\section{Bibliographie}

\section{Euvres étudiées}

Morency, Pierre. À l'heure du loup. Montréal: Boréal, 2002.

---, La Vie entière. Histoires naturelles du Nouveau Monde. Montréal: Boréal, 1996.

---, Les Paroles qui marchent dans la nuit. Montréal: Boréal, 1994.

---, L'œil américain. Histoires naturelles du Nouveau Monde. Montréal: Boréal, 1989.

---, «Effets personnels ». 1986. Poèmes 1966-1986. Montréal: Boréal, 2004: 249-74.

---, « Douze jours dans une nuit ». 1985. Poèmes 1966-1986. Montréal: Boréal, 2004: 233-48.

---, « Torrentiel ». 1978. Poèmes 1966-1986. Montréal: Boréal, 2004: 193-231.

---, «Lieu de naissance ». 1973. Poèmes 1966-1986. Montréal: Boréal, 2004: 161-91.

Nepveu, Pierre. Lignes aériennes. Montréal: Éditions du Noroît, 2002.

---, Romans-fleuves. Montréal: Éditions du Noroît, 1997.

Simard-Laflamme, Carole. Habit Habitat Habitus. Trois-Rivières: Le Sabord, 2002.

\section{Ouvrages critiques}

Barthes, Roland. «Littérature et discontinu». [Critique, 1962]. Essais critiques. Paris: Seuil, Coll. «Tel quel », 1964: 175-87.

Derrida, Jacques et Dufourmantelle, Anne. De l'hospitalité. Anne Dufourmantelle invite Jacques Derrida à répondre. Paris: Calmann-Lévy, Coll. « Petite bibliothèque des idées », 1997.

Foucault, Michel. «Le langage et l'espace ». [Critique 203 (avril 1964): 378-82]. Dits et Écrits. 1, 1954-1969. Dir. Daniel Defert et François Ewald. Paris: Gallimard, 1994 : 407-12.

Heidegger, Martin. "Bâtir Habiter Penser». [Vorträge und Aufsätze 1954]. Essais et conférences. Trad. par André Préau et préfacé par Jean Beaufret. Paris: Gallimard, Coll. « Tel $52 », 1958:$ 170-93.

${ }^{1}$ Nous n'employons pas ce terme dans le sens usuel d'apprivoiser un animal, de le rendre inoffensif et familier, et encore moins dans son sens analogique qui réfère à un processus d'assujettissement, d'asservissement. Nous 
expurgeons ici le processus de "domestication" de cette violence corollaire afin de ne retenir que le processus de rendre familier, de rapporter à l'image de la maison (domus).

${ }^{2} \mathrm{Cf}$. le langage de la théorie lorsqu'il réfère métaphoriquement à une « grammaire de l'action » par exemple. On en trouve un autre exemple dans L'Invention du quotidien de Michel de Certeau, où le langage sert de métaphore à l'espace : «L'espace serait au lieu ce que devient le mot quand il est parlé, c'est-à-dire quand il est saisi dans l'ambiguïté d'une effectuation. » (Certeau, Michel de. L'Invention du quotidien. Ed.10/18. Paris: Union Générale d'Éditions, $1980: 208$ ). 